\title{
The National Weather Service Ceilometer Planetary Boundary Layer Project
}

\author{
M. Hicks ${ }^{1}$, D. Atkinson ${ }^{1}$, B. Demoz ${ }^{3}$, K. Vermeesch ${ }^{2,3}$, R. Delgado ${ }^{3}$ \\ ${ }^{1} N W S$, Silver Spring, MD, USA; ${ }^{2} N A S A / G S F C$, Greenbelt, MD, USA; ${ }^{3} U M B C$, Baltimore, MD, USA
}

\begin{abstract}
The National Weather Service (NWS) is investigating the potential of utilizing the Automatic Surface Observing System's (ASOS) cloud base height indicator, the Vaisala CL31 ceilometer, to profile aerosols in the atmosphere. Field test sites of stand-alone CL31 ceilometers have been established, primarily, around the Washington DC metropolitan area, with additional systems in southwest USA and Puerto Rico. The CL31 PBL project examines the CL31 data collected for data quality, mixing height retrieval applicability, and its compliment to satellite data. This paper reviews the topics of the CL31 data quality and mixing height retrieval applicability.
\end{abstract}

\section{INTRODUCTION}

The National Weather Service (NWS) along with the Federal Aviation Administration (FAA) and the Department of Defense (DOD) owns an extensive suite of Automatic Surface Observing System (ASOS) stations. These stations span the USA with close to 1,000 sites and monitor atmospheric surface conditions (temperature, dewpoint, wind, etc.) and cloud base levels. The NWS, FAA and DOD are in the process of updating acquisition hardware and software for these stations as part of a Service Life Extension Program (SLEP). In this regard, the NWS Office of Science and Technology (OS\&T) and Office of Operational Systems (OOS) are investigating the potential of adding functionality to the ASOS cloud base height indicator, the Vaisala CL31 ceilometer.

The OS\&T NextGen Program and the OOS Sterling Field Support Center (SFSC) are collaborating with the NOAA Center of Atmospheric Sciences (NCAS) - Howard University Beltsville, MD Research Campus (HUBC), and University of Maryland Baltimore County (UMBC) to investigate the CL31 applicability to deriving boundary layer mixing heights and its compliment to satellite data. This project is known as the CL31 PBL Project. Of these topics, this paper discusses a review of status of the project and evaluation of the CL31 data quality and mixing height applicability.

\section{METHODOLOGY}

The CL31 PBL Project team has established data collection points in several locations: (1) on the west and northeast of Washington, DC metropolitan area in Sterling, VA (SFSC), College Park, MD (NCWCP), and Beltsville, MD (HUBC) since March 2013; (2) southwest Baltimore, MD at UMBC since October 2014; (3) University of Puerto Rico in Mayaguez since October 2014; and (4) west Texas alone Mexico's border at the University of El Paso since February 2015. The CL31 systems collect data at a $15 \mathrm{~s}$ frequency with a spatial resolution of $20 \mathrm{~m}$ from surface to $7.5 \mathrm{~km}$.

LIDARs (Light Detecting and Ranging) and radiosoundings are used to evaluate the CL31's data quality and retrieved mixing height products, respectively. At SFSC, HUBC and UMBC micropulse LIDARs (MPL) are used to evaluate the CL31's data quality. HUBC also has a newer generation Vaisala ceilometer, CL51, used to further evaluate the CL31's data quality and Vaisala BL-View mixing height software. The MPL's normalized relative backscatter (NRB) profile data is used to build a relation with the CL31's backscattered intensity; in addition, their profile gradient intensities are related (Campbell et al. 2002).

The SFSC, HUBC, and UMBC sites facilitate routine radiosonde soundings. Their datasets are used to evaluate LIDAR mixing height algorithms applied to CL31 profile data. The radiosondebased mixing heights are derived using the Bulk Richardson method. In addition, the mixing height comparison results are categorized by stability conditions; with the mean surface Bulk Richardson values (below $0.2 \mathrm{~km}$ ) less than -1 indicating unstable conditions and those greater or equal to -1 stable conditions. 


\section{RESULTS}

This section includes preliminary results of this ongoing study. So far, the collected CL31 data at SFSC have been qualitatively compared to that of the MPL. All major atmospheric features sensed by the MPL system were sensed with the CL31 system, up to $7.5 \mathrm{~km}$, as shown in Figure 1. More quantitative comparisons are currently being conducted. For example, the height of the gradient maximum and minimum up to $7.5 \mathrm{~km}$ in $3 \mathrm{hr}$ averaged backscatter profile periods are being correlated between the two systems. In addition, the difference of daily averaged NRB to that of the backscatter intensity of the CL31 is being correlated. This qualitative and quantitative analysis will be conducted at all LIDAR hosting data collection sites.

The CL31 PBL Project team has also evaluated Compton et al. 2013, Hicks et al. 2015 and Vaisala BL-View mixing height algorithms. So far, this analysis has been done for SFSC and HUBC. These algorithms, relative to radiosondebased derivations, showed the CL31 to be capable of detecting mixing heights, with a bias ranging from $-176 \mathrm{~m}$ to $527 \mathrm{~m}$ for unstable atmospheric conditions and $173 \mathrm{~m}$ to $1158 \mathrm{~m}$ for stable atmospheric conditions.
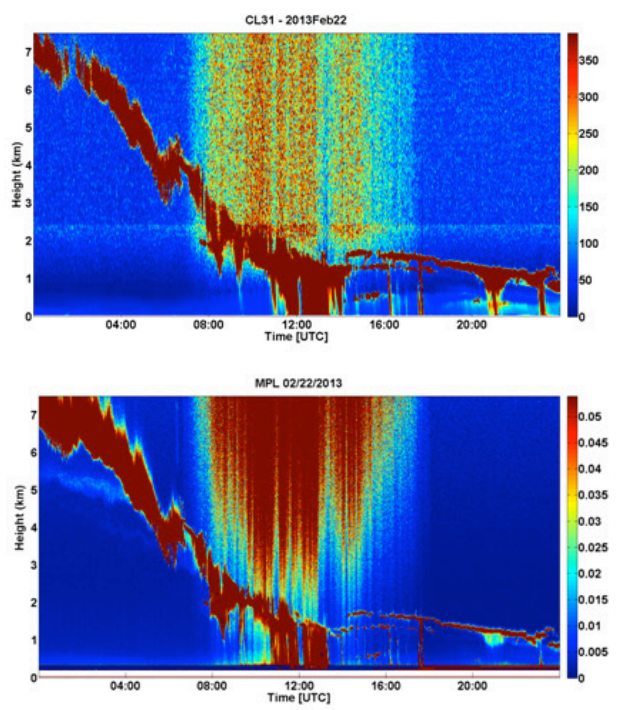

Figure 1. Comparison of time series of CL31 backscatter observations in top panel and MPL NRB observations in bottom panel at SFSC on February 22, 2013.

\section{CONCLUSION}

The CL31 ceilometer has a strong potential to be used for more than detecting cloud base heights in the ASOS network of almost 1000 stations across the USA. The profile data from these systems reflect real atmospheric features including frontal passages, low level jets, volcanic ash, smoke, thin clouds, and pollutants. In addition, the CL31 allows for the observation of mixing height which is lacking operationally.

The CL31 PBL team is pleased with the preliminary results and look forward to completing the analysis. The final goal of this project will be to provide concrete information on the impact of the CL31 profile data on volcanic ash, frontal passage, pollutant dispersion, and bay breeze monitoring/tracking and its overall impact on weather and dispersion forecast modeling.

\section{REFERENCES}

[1] Campbell, J. R., D. L. Hlavka, E. J. Welton, C. J. Flynn, D. D. Turner, J. D. Spinhirne, V. S. Scott III, I. H. Hwang, 2002: Full-time, Eye-Safe Cloud and Aerosol Lidar Observation at Atmospheric Radiation Measurement Program Sites: Instruments and Data Processing, J. Atmos. Ocean Tech., 19, 431-442.

[2] Compton, J. C., R. Delgado, T. A. Berkoff, R. M. Hoff, 2013: Determination of planetary boundary layer height on short spatial and temporal scales: A demonstration of the Covariance Wavelet Transform in ground based wind profiler and lidar measurements, J. Atmos. Ocean Tech., 30, 1566-1575.

[3] Hicks, M., S. Ricardo, E. Joseph, 2015: The Evaluation of a New Atmospheric Boundary Layer (ABL) Height Detection Method for Light Detecting and Ranging (LIDAR) Observations, Manuscript submitted for publication, J. Atmos. Ocean Tech., Manuscript submitted for publication. 